These data suggest that the extra sensitivity provided by AF bronchoscopy improves the ability of trainees to make the correct diagnosis at bronchoscopy and suggest a role for AFI in teaching hospitals in training inexperienced bronchoscopists.

Our preliminary study shows how a "slide bank" of bronchoscopic images can be used to asses the diagnostic performance of a bronchoscopic system by multiple operators. We believe it is valid and eminently transferable to other studies. This work needs to be repeated with a larger sample of patients and in a population with a higher incidence of intra-epithelial neoplasia.

\section{E.J. Cetti*, A.G. Nicholson", S. Singh", A.U. Wells* and P.L. Shah*,}

Depts of *Respiratory Medicine and "Histopathology, Royal Brompton Hospital, and "Dept of Respiratory Medicine, Chelsea and Westminster Hospital, London, UK.

Correspondence: P.L. Shah, Dept of Respiratory Medicine, Royal Brompton Hospital, Sydney Street, London, SW3 6NP, UK. E-mail: pallav.shah@imperial.ac.uk

Statement of Interest: Statements of interest for E.J. Cetti, A.U. Wells and P.L. Shah can be found at www.erj.ersjournals. $\mathrm{com} / \mathrm{misc} /$ statements.dtl

\section{REFERENCES}

1 Lam S, Kennedy $\mathrm{T}$, Unger $\mathrm{M}$, et al. Localization of bronchial intraepithelial neoplastic lesions by fluorescence bronchoscopy. Chest 1998; 113: 696-702.

2 Ikeda N, Honda H, Hayashi A, et al. Early detection of bronchial lesions using newly developed videoendoscopy-based autofluorescence bronchoscopy. Lung Cancer 2006; 52: 21-27.

3 Chiyo M, Shibuya K, Hoshino H, et al. Effective detection of bronchial preinvasive lesions by a new autofluorescence imaging bronchovideoscope system. Lung Cancer 2005; 48: 307-313.

4 Travis WD, Brambilla E, Muller-Hermelink HK, eds. In: World Health Organization Classification of Tumours. Pathology and Genetics: Tumours of the Lung, Pleura, Thymus And Heart. Lyon, IARC Press, 2004.

5 Lam S, Kennedy T, Unger M, et al. Localization of bronchial intraepithelial neoplastic lesions by fluorescence bronchoscopy. Chest 1998; 113: 696-702.

6 Vermylen P, Pierard P, Roufosse C, et al. Detection of bronchial preneoplastic lesions and early lung cancer with fluorescence bronchoscopy: a study about its ambulatory feasibility under local anaesthesis. Lung Cancer 1999; 25: 161-168.

7 Ernst A, Simoff MJ, Mathur PN, et al. D-light autofluorescence in the detection of premalignant airway changes. a multicenter trial J Bronchol 2005; 12: 133-138.

8 Aziz ZA, Wells AU, Hansell DM, et al. HRCT diagnosis of diffuse parenchymal lung disease: interobserver variation. Thorax 2004; 59: 506-511.

\title{
Assaying for circulating osteopontin in practice: a technical note
}

\section{To the Editors:}

Plasma osteopontin is a very interesting marker for pulmonary diseases, being proposed for evaluating prognosis in patients with nonsmall cell lung cancer [1] or tuberculosis [2]. Osteopontin has also been implicated in the pathogenesis of idiopathic pulmonary fibrosis [3] and chronic obstructive pulmonary disease [4]. A more debated use of this marker has been its diagnostic utility in malignant pleural mesothelioma [5, 6]. Two major commercial kits are available, supplied by R\&D systems, Minneapolis, MN, USA (Quantikine Human Osteopontin Immunoassay) and by Immuno-Biological Laboratories Co., Ltd, Gunma, Japan (Human osteopontin ELISA). It has been suggested in the literature that the results of osteopontin dosage are highly dependent on the choice of the ELISA kit. Indeed a previous publication reported important differences between available assays with little correlation between the results of the two commercial kits [7]. However, correlation is not the only analysis that should be performed when comparing two tests that measure the same variable [8].

We performed a comparative evaluation of the two commercial ELISA kits in a series of 75 samples of plasma retrieved from 13 patients treated for tuberculosis. The samples were obtained at diagnosis, after 1, 2, 4 and 8 weeks, and 4 and 6 months of treatment. A standard operation procedure was in place for retrieving the samples and all patients gave informed consent. After centrifugation, plasma samples were immediately transferred in a $-80^{\circ} \mathrm{C}$ freezer until assayed. A mean of six samples per patient (interquartile range 5-6) were available.

A correlation analysis between the two measurements yielded a Spearman $\rho$ of only 0.4924 but this was statistically significant ( $p<0.001$; fig. 1a). If we used the Pearson correlation coefficient (and thus assuming a normal distribution of data) this correlation was also low but statistically significant $(\mathrm{r}=0.4515 ; \mathrm{p}<0.001)$. However, when we used a Bland-Altman plot to analyse the results, we obtained an almost straight line (fig. 1b). Thus, the lack of strong correlation between the two methods is, in fact, the result of a systematic bias between the two methods, which is proportional with the measured value. This type of result, called "proportional error" is, in fact, the result of the nonlinearity of the two measures and probably is a consequence of the amplification generated by different enzymes in the ELISA assay. Since we do not have a "gold standard", we cannot say exactly which method is biased and 

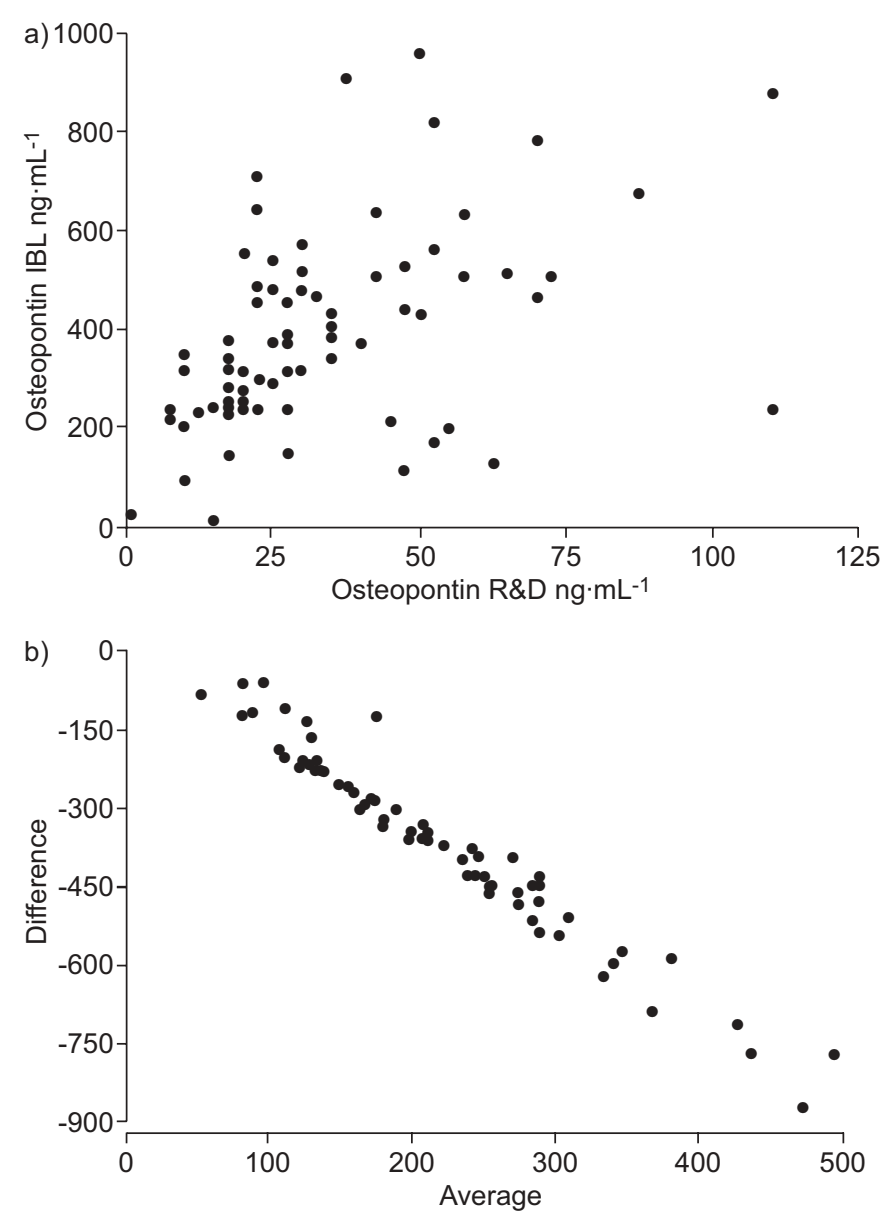

FIGURE 1. a) Correlation between the values obtained for osteopontin in plasma assayed with the Quantikine Human Osteopontin Immunoassay (R\&D systems, Minneapolis, MN, USA) (R\&D) and the Human osteopontin ELISA (Immuno-Biological Laboratories Co., Ltd, Gunma, Japan) (IBL). Spearman $\rho=0.4924$. b) Bland-Altman plot of the measurements for osteopontin showing a strong proportional error between the two methods.

in which direction. Mainly, both assays measure the same target molecule despite using different antibodies. These data must be taken into account when interpreting and comparing the results between different publications as well as choosing an assay for use in practice.
D. Constantinescu*, M. Vornicu" , C. Grigoriu ", C. Cozmei* and B.D. Grigoriu ${ }^{\#+}$

*Institute of Public Health, "Clinic of Pulmonary Diseases, "Depts of Forensic Pathology, and ${ }^{+}$Dept of Pulmonary Diseases, University of Medicine and Pharmacy "Gr. T. Popa", Iasi, Romania.

Correspondence: B.D. Grigoriu, Dept of Pulmonary Diseases, University of Medicine and Pharmacy "Gr. T. Popa" Iasi, Str Universitatii 16, 700115 Iasi, Romania. E-mail: b_grigoriu@ hotmail.com

Support Statement: This research has been funded by the National Council for Scientific Research in Higher Education (CNCSIS) by the grant number 1191/2007.

Statement of Interest: None declared.

\section{REFERENCES}

1 Isa S-I, Kawaguchi T, Teramukai S, et al. Serum osteopontin levels are highly prognostic for survival in advanced non-small cell lung cancer: results from JMTO LC 0004. J Thorac Oncol 2009; 4: 1104-1110.

2 Inomata S, Shijubo N, Kon S, et al. Circulating interleukin-18 and osteopontin are useful to evaluate disease activity in patients with tuberculosis. Cytokine 2005; 30: 203-211.

3 Pardo A, Gibson K, Cisneros J, et al. Up-regulation and profibrotic role of osteopontin in human idiopathic pulmonary fibrosis. PLoS Med 2005; 2: e251.

4 Chung KF, Adcock IM. Multifaceted mechanisms in COPD: inflammation, immunity, and tissue repair and destruction. Eur Respir J 2008; 31: 1334-1356.

5 Scherpereel A, Astoul P, Baas P, et al. Guidelines of the European Respiratory Society and the European Society of Thoracic Surgeons for management of malignant pleural mesothelioma. Eur Respir J 2010; 35: 479-495.

6 Creaney J, Yeoman D, Demelker Y, et al. Comparison of osteopontin, megakaryocyte potentiating factor, and mesothelin proteins as markers in the serum of patients with malignant mesothelioma. J Thorac Oncol 2008; 3: 851-857.

7 Vordermark D, Said HM, Katzer A, et al. Plasma osteopontin levels in patients with head and neck cancer and cervix cancer are critically dependent on the choice of ELISA system. BMC Cancer 2006; 6: 207.

8 Bland JM, Altman DG. Statistical methods for assessing agreement between two methods of clinical measurement. Lancet 1986; 1: 307-310.

DOI: $10.1183 / 09031936.00160609$

\section{Venoatrial compression by lymphadenopathy in sarcoidosis}

To the Editors:

Thoracic lymphadenopathy in sarcoidosis usually displays characteristic and well-known features, such as a symmetrical hilar distribution and a noncompressive nature. However, compressive lymphadenopathy is known as an atypical yet possible presentation of the disease. Several case reports and reviews have previously reported compressions of lobar or segmental bronchi, pulmonary artery branches, superior vena cava or the left laryngeal recurrent nerve [1-4]. To the best of 